\title{
Broadband pump-probe imaging spectroscopy applicable to ultrafast single-shot events
}

Yasuo Minami, Hiromoto Yamaki, Ikufumi Katayama, and Jun Takeda*

Department of Physics, Graduate School of Engineering, Yokohama National University, Yokohama 240-8501, Japan

E-mail: jun@ynu.ac.jp

(Received November 7, 2013; accepted December 18, 2013; published online January 15, 2014)

We propose a scheme for frequency-resolved single-shot spectroscopy with an echelon mirror. The echelon mirror is employed to generate spatially encoded time delays for the white-light continuum probe beam; it produces a temporal step of $66 \mathrm{fs}$ and an overall time delay of $33 \mathrm{ps}$. We demonstrate broadband pump-probe imaging spectroscopy and present time-frequency two-dimensional images of the transient absorption of $\beta$-carotene between 420 and $630 \mathrm{~nm}$ with single-shot detection. The results show that this technique is a powerful tool for observing the ultrafast, broadband transient dynamics of materials that exhibit irreversible reactions or deterioration by laser pulse irradiation. 
Various spectroscopic techniques to reveal the ultrafast dynamics of materials have been developed since the invention of ultrashort-pulsed lasers. One of the most common techniques is pump-probe spectroscopy, which reveals various ultrafast phenomena such as excited state dynamics, phonon dynamics, and photochemical reactions. ${ }^{1-5)}$ This technique, however, requires that the pump-probe sequence be repeated multiple times to obtain information in the temporal and spectral regions of interest; therefore, it is unsuitable for samples showing either irreversible reactions or deterioration induced by intense pump pulses. To address these limitations, a number of spectroscopic techniques based on single-shot detection have been proposed. ${ }^{6-14)}$ Single-shot techniques are usually realized by manipulating the probe pulse to produce a spatially encoded time delay, but this presents some experimental difficulties: in the dual-echelon technique, only the temporal profiles of the transient signals can be obtained, ${ }^{6-8)}$ whereas in pump-probe imaging spectroscopy, a large spot size at the sample is needed to detect transient signals having a wide temporal range. ${ }^{9-11)}$ Thus, none of these techniques can measure transient signals with wide temporal and spectral ranges.

We recently proposed a time-frequency two-dimensional (2D) pump-probe imaging spectroscopy with an echelon mirror based on single-shot detection in an effort to remove these constraints. ${ }^{12-14)}$ One of the technical merits of our imaging spectroscopy is the adjustability of the echelon mirror; it can be designed with a desirable step-width and step-height, which enables us to observe various transient phenomena of interest with a suitable time resolution, overall time delay, and spectral bandwidth. In past works, we applied this method to the visualization of phonon-polariton oscillations in ferroelectric crystals, ${ }^{12)}$ an autocorrelation system, ${ }^{13)}$ and terahertz wave detection. ${ }^{14)}$ Here, we design an echelon mirror applicable to wider spectral and temporal ranges of 420-630 nm and a few tens of picoseconds, respectively, and demonstrate broadband single-shot pump-probe spectroscopy. Using our method, we can observe the real-time absorption changes of a liquid solution with a broadband spectral region free from photodegradation. We present the results for $\beta$-carotene, a pigment that is fundamental to photosynthesis and has known ultrafast excited state dynamics. The transient absorption of $\beta$-carotene in the visible range is observed, ${ }^{15-21)}$ but a dynamical model of the pigment has not been completely clarified because it easily photodegrades under pump laser pulse illumination. ${ }^{9-11)}$ The optical properties of $\beta$-carotene have been measured in a rotating or flowing cell to avoid photodegradation. ${ }^{19,22)}$ Our imaging spectroscopy method employing an echelon mirror offers a single-shot technique and enables the true absorbance change to be estimated, so it may completely eliminate the effects of photodegradation and could be applied without the need for flowing specimens. 
The experimental system employed in this study is shown in Fig. 1(a). A Ti:sapphire amplifier system with a repetition frequency of $1 \mathrm{kHz}$ provides 150 -fs laser pulses with a center wavelength of $800 \mathrm{~nm}$. The pump pulse passes through a $\beta$-barium borate (BBO) crystal and generates pulses with a wavelength of $400 \mathrm{~nm}$, which are focused on the specimen with a cylindrical lens having a focal length of $150 \mathrm{~mm}$. The probe pulse is focused on a $\mathrm{CaF}_{2}$ crystal and generates white light by self-phase modulation. The white light is then directed toward the reflective echelon mirror with 500 steps at a step width and height of 80 and 10 $\mu \mathrm{m}$, respectively. The echelon mirror is fabricated on a nickel block using a precise micromachining technique and has a surface roughness of $<0.2 \mu \mathrm{m},{ }^{13)}$ which results in minimal spatiotemporal modification of the probe beam. The echelon mirror is used to produce multiple probe beamlets. The probe beam diffracted from the echelon mirror has a 66-fs time delay for each step and an overall time delay of $33 \mathrm{ps}$. The probe beam is focused to a spot on the specimen $1 \mathrm{~mm}$ in diameter that is also illuminated by the pump pulse with a slightly larger spot (1.2 $\mathrm{mm}$ in diameter) having homogeneous intensity. After passing through the sample, the probe beam is imaged onto the entrance slit of a spectrometer attached to a charge coupled device (CCD) detector (1024x1024 pixels). In this detection system, the broadband probe beam is spectrally dispersed by the echelon mirror. To suppress this dispersion and to image the probe beam precisely onto the entrance slit, the double $4 f$ ( 2 $f-2 f)$ configuration is applied to the optical setup using a concave mirror $\left(F_{1}=100 \mathrm{~mm}\right)$, a convex lens $\left(F_{2}=100 \mathrm{~mm}\right)$, a cylindrical lens $\left(F_{3}=250 \mathrm{~mm}\right)$, and an imaging lens $\left(F_{4}=50\right.$ $\mathrm{mm})$, as shown in Fig. 1(b); the concave mirror suppresses the chromatic aberration and the chirp effect of the white light, which is important for broadband time-resolved spectroscopy.

To calibrate the temporal axis, we used the optical delay stage set in the pump arm and measured time-frequency images of the Kerr signal of the white light continuum with different time delays. The Kerr signal moved linearly along the temporal axis of the CCD detector, and the obtained calibration factor was $50.5 \mathrm{fs} /$ pixel, indicating that the temporal axis is not seriously distorted by the use of the concave mirror with an off-axis configuration. We used the calibration factor for all the measurements. The raw 2D image showed the chirp characteristics due to the group-velocity dispersion of the probe-beam path; therefore, we also adjusted the temporal axis by a factor derived from fitting of the Kerr signal by Cauchy's dispersion equation. ${ }^{10,23)}$ In these calibrations, we used $\mathrm{CS}_{2}$ as the Kerr gate medium. ${ }^{20)}$ The time resolution of the spectroscopy system is sufficiently high compared to the time constant of the absorbance changes of the specimen. The response time of our system was estimated to be 200 fs using optical Kerr gate signals of the white light continuum, where a quartz plate served as the Kerr medium. ${ }^{20)}$ Using our double $4 f$ configuration, a step terrace of the echelon 
mirror $(80 \mu \mathrm{m}$ width) is imaged to $<2$ pixels of the CCD detector, yielding a resolution of $<101 \mathrm{fs}$. This value is less than the observed Kerr response; therefore, the time resolution of the spectroscopy system is determined to be $200 \mathrm{fs}$.

As a demonstration, a commercial powder of $\beta$-carotene (Wako Pure Chemical Industries, special grade) was used without further purification and was dissolved in $n$-hexane with a concentration of $1.2 \times 10^{-4} \mathrm{~mol} / \mathrm{l}$. Figure 2(a) shows a transient absorption image of the $\beta$-carotene solution measured using the single-shot technique. The absorbance change [i.e., the change in the optical density $(\Delta \mathrm{OD})]$ is indicated on a color scale, and the time delay is that between the pump and probe pulses. As the 420-630-nm-wavelength white pulse is observed on the CCD camera within a single shot, transient bleaching at 430-500 nm and transient absorption at 520-630 $\mathrm{nm}$ were mapped over wide temporal and spectral regions of about 20 ps and 420-630 nm, respectively. The time span is limited by the signal-to-noise ratio and the intensity distribution of the probe pulse. The absorption saturation (bleaching) originates from the transition from the $1^{1} \mathrm{~A}_{\mathrm{g}}{ }^{-}$state to the $1^{1} \mathrm{~B}_{\mathrm{u}}{ }^{+}$state of $\beta$-carotene, whereas stimulated absorption in the transition from the metastable state of the $2^{1} \mathrm{~A}_{\mathrm{g}}{ }^{-}$state to the higher order states is responsible for the transient absorption. ${ }^{18)}$

Figure 2(b) shows temporal traces of the transient absorption at $470 \mathrm{~nm}$ (lower curve; blue) and $560 \mathrm{~nm}$ (upper curve with a vertical offset; red) taken from Fig. 2(a). Here, we phenomenologically estimated the optical response of the rise time $\tau_{1}$ and decay time $\tau_{2}$ using

$$
\alpha(t)=\alpha_{0}\left[1-\exp \left(-\frac{t}{\tau_{1}}\right)\right] \times \exp \left(-\frac{t}{\tau_{2}}\right)
$$

where $\alpha_{0}$ is the absorption constant. Equation (1) is convoluted with the time profile of the laser pulse and fitted to the obtained time profile of the absorption change. The dashed curves in Fig. 2(b) indicate best fits to the experimental data using Eq. (1) with $\tau_{1}=0.40$ and $\tau_{2}=8.5$ ps for the $560 \mathrm{~nm}$ trace, and $\tau_{1}=0.33$ and $\tau_{2}=7.8 \mathrm{ps}$ for the $470 \mathrm{~nm}$ trace, which are almost in agreement with the previously reported values. ${ }^{5,24}$ Figure 3 shows the steady-state absorption spectrum and temporal absorption spectra for time delays of $0-15$ ps obtained by taking profiles of the data in Fig. 2(a). In the short-wavelength range, the characteristics of bleaching, which are not observed in steady-state spectroscopy, ${ }^{19)}$ appear.

Because our method can measure the absorbance change in a single shot, it is possible to evaluate the effects of photodegradation. Figure 4 shows the dependence of the photoinduced absorption change on the number of pump shots for a wavelength of $560 \mathrm{~nm}$. We can see that the absorbance change is sensitive to the number of pump pulses in the few pulse limit but 
saturates in the many-pulse limit. From the best-fit curve, the true absorbance change is estimated to be 0.24 , which corresponds well to the value obtained by our single-shot system in this study and that obtained by the accumulation of single-shot data. ${ }^{11)}$ The derived relaxation shot number is 7.6 shots, which means that the specimen is completely photodegraded after 7.6 shots. The rapid decrease in the absorbance change indicates explicitly that photodegradation-free observation of the specimen is achieved only in the single-shot measurement. The single-shot imaging method thus suppresses the effects of photodegradation and enables a true measurement of the response to light.

In conclusion, we developed a single-shot optical pump-probe spectroscopy system that is applicable to the spectral region of $420-630 \mathrm{~nm}$ and a temporal range of about $20 \mathrm{ps}$. We demonstrated pump-probe imaging of a $\beta$-carotene solution with the system and obtained the absorption change in the specimen. These results show that our broadband pump-probe imaging spectroscopy can be used to measure the transient behavior of various specimens over wide spectral and temporal ranges, such as photochromic reactions, photoresponses of a tiny amount of solution, or ultrafast dynamics over the photo-damage threshold.

\section{Acknowledgment}

This work was supported in part by Grants-in-Aid for Scientific Research (KAKENHI, Nos. 23104713, 23241034, and 25104712) from the Japan Society for the Promotion of Science. 


\section{References}

1) M. Drescher, M. Hentschel, R. Kienberger, M. Uiberacker, V. Yakovlev, A. Scrinzi, T. Westerwalbesloh, U. Kleineberg, U. Heinzmann, and F. Krausz, Nature 419, 803 (2002).

2) S. Woutersen, U. Emmerichs, and H. J. Bakker, Science 278, 658 (1997).

3) R. W. Schoenlein, J. G. Fujimoto, G. L. Eesley, and T. W. Capehart, Phys. Rev. B 43, 4688 (1991).

4) Y. J. Yan and S. Mukamel, Phys. Rev. A 41, 6485 (1990).

5) W. B. Bosma, Y. J. Yan, and S. Mukamel, J. Chem. Phys. 93, 3863 (1990).

6) K. Y. Kim, B. Yellampalle, A. J. Taylor, G. Rodriguez, and J. H. Glownia, Opt. Lett. 32, 1968 (2007).

7) G. P. Wakeham, D. D. Chung, and K. A. Nelson, Thermochim. Acta 384, 7 (2002).

8) G. P. Wakeham and K. A. Nelson, Opt. Lett. 25, 505 (2000).

9) J. Takeda, A. Ishida, Y. Makishima, and I. Katayama, Sensors 10, 4253 (2010).

10) Y. Makishima, N. Furukawa, A. Ishida, and J. Takeda, Jpn. J. Appl. Phys. 45, 5986 (2006).

11) N. Furukawa, C. M. Mair, V. D. Kleiman, and J. Takeda, Appl. Phys. Lett. 85, 4645 (2004).

12) H. Sakaibara, Y. Ikegaya, I. Katayama, and J. Takeda, Opt. Lett. 37, 1118 (2012).

13) I. Katayama, H. Sakaibara, and J. Takeda, Jpn. J. Appl. Phys. 50, 102701 (2011).

14) Y. Minami, Y. Hayashi, J. Takeda, and I. Katayama, Appl. Phys. Lett. 103, 051103 (2013).

15) M. G. Vivas, D. L. Silva, L. de Boni, R. Zalesny, W. Bartkowiak, and C. R. Mendonca, J. Appl. Phys. 109, 103529 (2011).

16) D. Kosumi, M. Komukai, H. Hashimoto, and M. Yoshizawa, Phys. Rev. Lett. 95, 213601 (2006).

17) D. Polli, G. Cerullo, G. Lanzani, S. De Silvestri, K. Yanagi, H. Hashimoto, and R. J. Cogdell, Phys. Rev. Lett. 93, 163002 (2004).

18) N. Furukawa and J. Takeda, Nonlinear Opt. 29, 579 (2002).

19) M. Yoshizawa, H. Aoki, and H. Hashimoto, Phys. Rev. B 63, 180301(R) (2001).

20) J. Takeda, K. Nakajima, S. Kurita, S. Tomimoto, S. Saito, and T. Suemoto, Phys. Rev. B 62, 10083 (2000).

21) T. Sashima, Y. Koyama, T. Yamada, and H. Hashimoto, J. Phys. Chem. B 104, 5011 (2000). 
22) J.-P. Zhang, R. Fujii, Y. Koyama, F. S. Rondonuwu, Y. Watanabe, A. Mortensen, and L. H. Skibsted, Chem. Phys. Lett. 348, 235 (2001).

23) E. Hecht, Optics (Addison-Wesley, Redwood City, MA, 2001) 4th ed., Chap. 3.

24) D. Kosumi, K. Yanagi, T. Nishio, H. Hashimoto, and M. Yoshizawa, Chem. Phys. Lett. 408,89 (2005). 


\section{Figure Captions}

Fig. 1. (a) Overview of experimental setup. (b) Optical paths of probe and pump pulses. White light generated at the $\mathrm{CaF}_{2}$ crystal is focused by the echelon mirror onto the specimen. The transmitted probe is then directed toward the spectrometer and CCD camera through a cylindrical lens and convex imaging lens. Aberrations are suppressed by a double $4 f$ configuration with a concave mirror, convex lens, cylindrical lens, and convex imaging lens. The CCD camera records a time-resolved spectrum.

Fig. 2. (a) Transient absorption imaging of $\beta$-carotene solution using single-shot spectroscopy. The absorbance change is indicated on a color scale, and the time delay is that between the pump and probe pulses. (b) Temporal traces of the transient absorption at $470 \mathrm{~nm}$ (lower; blue) and $560 \mathrm{~nm}$ (upper, with offset of +0.1 ; red) taken from (a).

Fig. 3. (a) Steady-state absorption spectrum and (b) temporal absorption spectra obtained from profiles of the data in Fig. 2(a).

Fig. 4. Dependence of the photoinduced absorption change on the number of pump shots. Dashed curve is best-fit single exponential decay with an offset. 
(a)

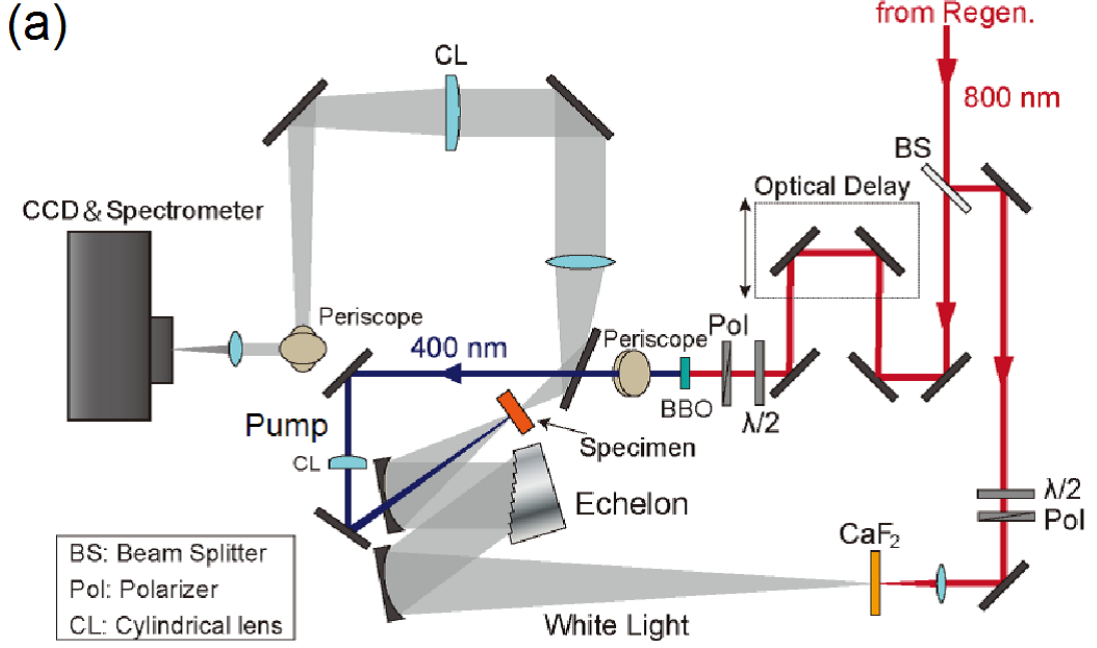

(b)

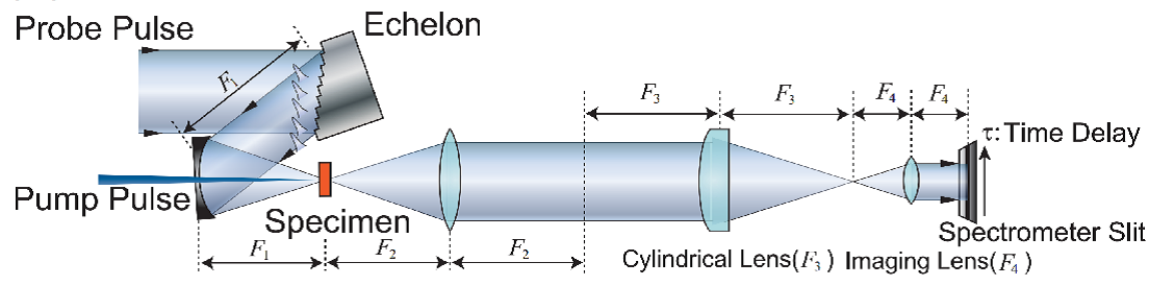

Fig. 1 


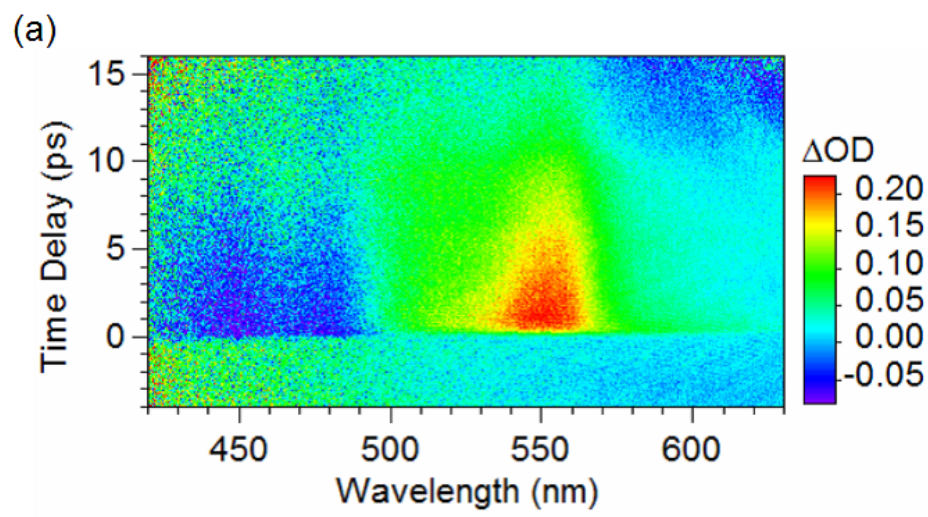

(b)

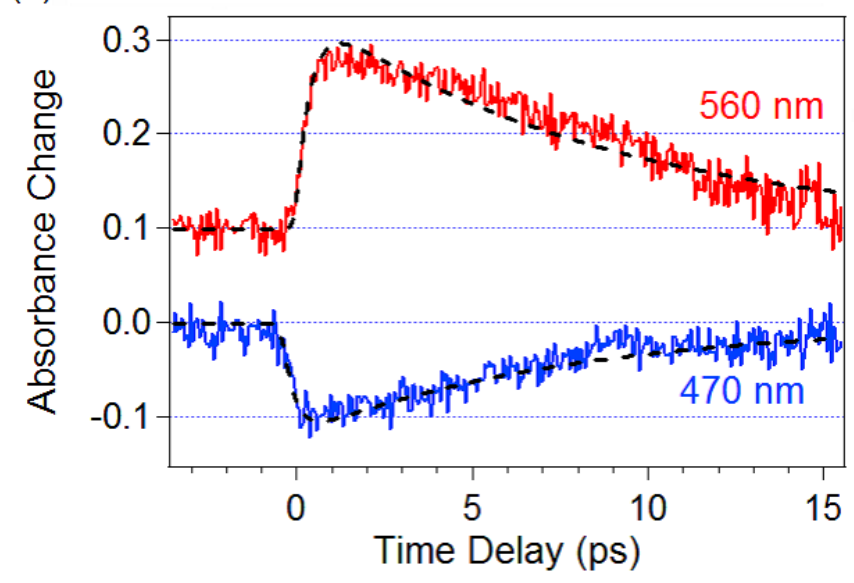

Fig. 2 


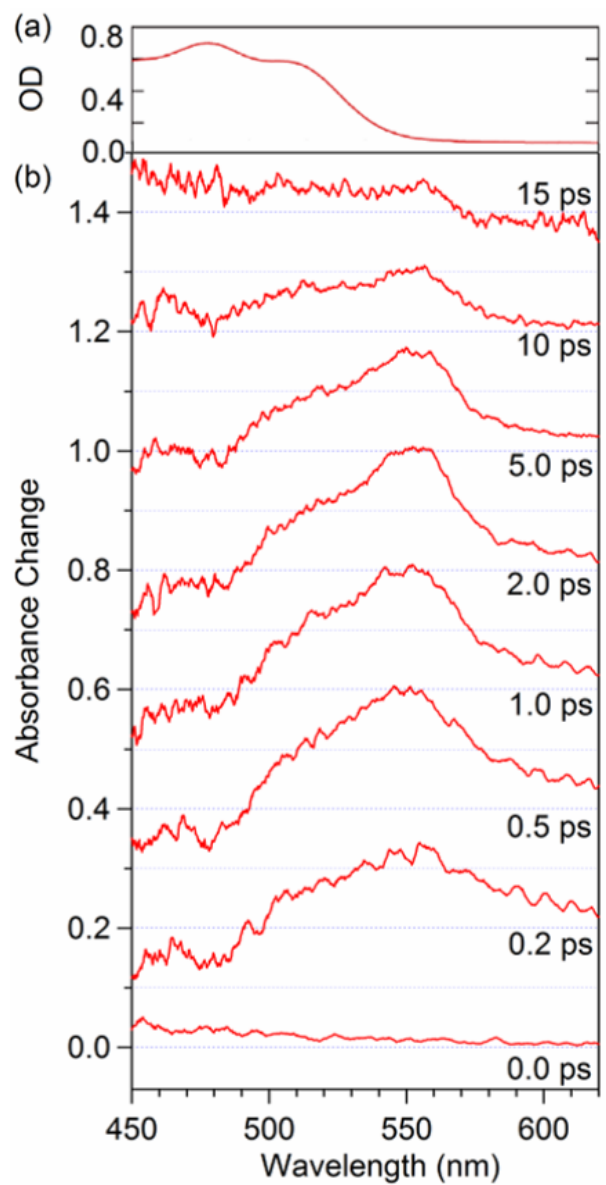

Fig. 3 


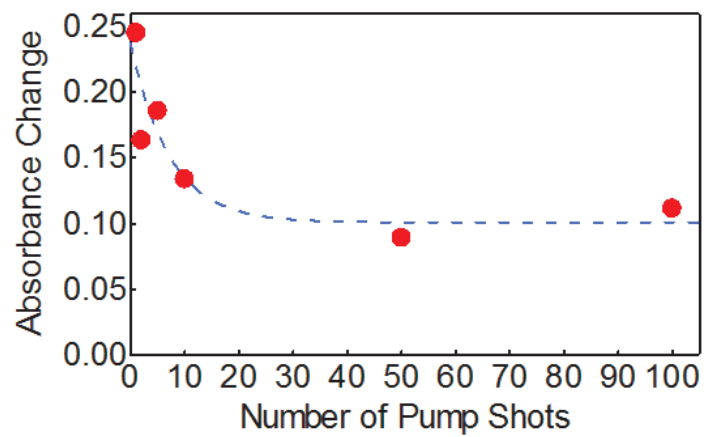

Fig. 4 